\title{
Acceso a cuidados de salud: discursos bioéticos del Norte y del Sur
}

\author{
Access to healthcare: bioethical discourses from North and South
}

Plinio José Cavalcante Monteiro', Camilo Hernán Manchola Castillo²

DOI: 10.1590/0103-110420195509

RESUMEN Estudio comparativo adoptando la técnica de investigación bibliográfica, mediante identificación y lectura de obras de referencia de consagrados autores de la bioética, con el objetivo de analizar discursos bioéticos sobre la temática del acceso a la salud en Estados Unidos y Brasil. Identificamos que el énfasis de los discursos estadounidenses está en la adquisición de bienes y servicios de salud y en estrategias para garantizar el acceso a seguros de salud, que es vista como una mercancía. Por el contrario, los discursos brasileños se centran en defender el derecho y acceso universal a la salud, que es vista como un valor social, un derecho humano fundamental. Los autores concluyen que los discursos bioéticos sobre el acceso a la salud encarnan diferentes perspectivas ético-políticas. En este sentido, las políticas de salud pueden promover - o no - los derechos humanos, incluyendo el derecho a la salud, dependiendo de cómo son concebidas o ejecutadas.

PALABRAS CLAVE Bioética. Salud pública. Accesibilidad a los servicios de salud.

ABSTRACT Comparative study, with a hermeneutical approach, adopting the bibliographic research technique, through identification and reading of reference works by renowned authors of bioethics, with the aim of analyzing bioethical discourses on the subject of access to healthcare in the United States and Brazil. We identify that the emphasis of American discourses is on the acquisition of health goods and services and on strategies to guarantee access to health insurance, which is seen as a commodity. On the contrary, Brazilian discourses focus on defending the right and universal access to health, which is seen as a social value, a fundamental human right. The authors conclude that bioethical discourses on access to health embody different ethical-political perspectives. In this sense, health policies may or may not promote human rights, including the right to healthcare, depending on how they are conceived or executed.

KEYWORDS Bioethics. Public health. Health services accessibility.

1 Universidade Federal do Amazonas (Ufam) -

Manaus (AM), Brasil.

2 Universidade de Brasília (UnB) - Brasília (DF),

Brasil. 


\section{Introducción}

El uso de los servicios de salud, mediante prestación de cuidados a los pacientes, representa la función central en el funcionamiento de los sistemas de salud. Los cuidados de salud prestados a los pacientes constituyen un aspecto fundamental del derecho a la salud y merecen atención como una cuestión relativa a los derechos humanos'. Proporcionar un adecuado acceso a servicios de salud es esencial para la efectividad en la prestación de este derecho.

En las últimas décadas se han propuesto diversos conceptos sobre acceso a los cuidados de salud $^{2}$. Estos conceptos consideran diferentes variables, desde las condiciones individuales de los usuarios de los servicios ${ }^{\mathbf{3}, 4}$, tales como aspectos económicos, educacionales, sociales y culturales, hasta la disponibilidad geográfica y organizacional del sistema de salud ${ }^{5}$, pasando por la libertad de los individuos para escoger y usar los servicios disponibles ${ }^{6,7}$. Los conceptos más actuales de acceso a los cuidados de salud incorporan varios de estos elementos ${ }^{8}$.

Se considera que un adecuado acceso a los cuidados de salud se torna efectivo cuando están disponibles los recursos de salud (disponibilidad), cuando se adecuan a las necesidades de los individuos y de las comunidades (adecuación), cuando pueden ser utilizados por los diferentes usuarios (goce) y resuelven de manera satisfactoria los diferentes problemas de salud (resolutividad).

El acceso a los cuidados de salud, aunque restringido al nivel de la asistencia en salud (prestación de servicios), es condición primordial para alcanzar la justicia distributiva y equidad en salud. Además, la equidad en el acceso a los servicios de salud es fuertemente influenciada por el modelo de asistencia adoptado en los diferentes sistemas de salud.

Los modelos contemporáneos de sistemas de salud parecen adecuados para este análisis sobre justicia distributiva y equidad en el acceso a los cuidados de salud, pues involucran importantes cuestiones éticas en escenarios con enormes complejidades económicas, políticas, sociales y culturales. De la misma manera, no siempre la mayor riqueza de una sociedad se traducirá obligatoriamente en justicia social, o sea, por sí misma no garantizará una justa y equitativa distribución de los recursos disponibles.

El objetivo de este estudio es analizar críticamente dos diferentes visiones sobre el tema de acceso a cuidados de salud: una liberal, con fuerte influencia del mercado como orientador del proceso de distribución de recursos sanitarios; y una social, con fundamento en los derechos humanos y una determinante participación del Estado en la elaboración y efectivación de las políticas públicas de salud. Finalmente, se buscará establecer una importante diferenciación ético-política e ideológica entre Sistema Universal de Salud (Universal Health System - UHS) y Cobertura Universal de Saúde (Universal Health Coverage - UHC).

Estudio comparativo adoptando la técnica de investigación bibliográfica, mediante identificación y lectura de las obras de referencia de consagrados autores de la bioética: Hugo Tristram Engelhardt Jr., Tom Beauchamp, James Childress, Norman Daniels, Miguel Kottow, Fermin Roland Schramm y Volnei Garrafa.

La elección de los referidos autores, en dos contextos geopolíticos distintos (Estados Unidos de América y Brasil), se dio con base en el contenido y la relevancia de sus producciones científicas para el tema de este estudio: el acceso a cuidados de salud.

\section{Los discursos bioéticos del norte}

El énfasis de los bioeticistas estadounidenses seleccionados radica en la adquisición de bienes y servicios de salud y en las estrategias para garantizar el acceso a los seguros de salud, vista como mercancía (commodity); como se verá, apenas es posible notar un posicionamiento discretamente discordante de Norman Daniels. 
Según Engelhardtª en su clásica obra 'Fundamentos de la Bioética', un sistema de asistencia a la salud que considere sus limitaciones morales y financieras precisa aceptar que las desigualdades en el acceso a la salud son moralmente inevitables y que los costos de los servicios de salud deben ser soportados por un sistema de salud económicamente eficiente; así, la existencia de desigualdades sociales no autoriza al uso de la fuerza contra los individuos que poseen los recursos con el objetivo de atender las necesidades de los desposeídos, pues tales limitaciones de recursos son circunstanciales y deben ser moralmente respetadas.

Engelhardt, en su obra 'Fundamentos de la Bioética Cristiana Ortodoxa'10, considera moralmente indefendible la excesiva intervención del Estado en los procesos de distribución de recursos para la salud, sobre todo cuando estos recursos son originalmente privados, debiendo limitarse a los recursos exclusivamente públicos. Así, sugiere como ideal un sistema de salud en capas - niveles de acceso a los cuidados de salud de acuerdo con la capacidad de pago de los individuos. Un sistema de salud de esta naturaleza representa un compromiso, pues proporciona alguna asistencia a la salud para casi todos y autoriza a los que disponen de recursos a que compren servicios adicionales o mejores.

Se identifica en Engelhardt un discurso ultraliberal, en el que los individuos pasan a ser vistos como clientes y en el que el mercado debe orientar, de acuerdo con las condiciones financieras de cada capa de la población, qué servicios pueden estar disponibles.

Beauchamp y Childress"1, al tratar del principio de justicia (Justice) en el capítulo 7 de la última edición de su libro 'Principles of Biomedical Ethics', defienden la importancia de discutir las desigualdades en el acceso a la atención sanitaria desde el punto de vista de la justicia, tanto a nivel local (particular societies) como global (global community). Sin embargo, reconocen que un serio problema con el acceso a la atención sanitaria consiste en determinar qué beneficios deben garantizarse, particularmente cuando se parte de la perspectiva de un derecho de acceso igualitario a los recursos de salud; es decir, aunque todas las personas tienen el derecho de no ser impedidas de obtener asistencia sanitaria, este derecho no garantiza que los demás deban suministrar bienes, servicios o recursos de salud.

Una interpretación literal de este derecho significa que todos, en cualquier lugar, deben tener acceso igualitario a todos los bienes y servicios disponibles para cualquier persona. En su lugar, Beauchamp y Childress defienden el derecho a un 'mínimo decente' (decent minimum) de atención en salud, un objetivo moderado e igualitario de acceso universal (al menos en una comunidad política) para los cuidados básicos de salud"1.

La concepción de Beauchamp y Childress es de un sistema de salud en dos niveles de asistencia: cobertura social reforzada para necesidades de salud básicas y catastróficas (nivel 1), y cobertura privada voluntaria para otras necesidades y deseos de salud (nivel 2). El primer nivel atiende las necesidades de salud a través del acceso universal a los servicios básicos; este nivel supuestamente cubre al menos protecciones de salud pública y atención preventiva, atención primaria, cuidados intensivos y servicios sociales especiales para las personas con discapacidad. En el segundo nivel, servicios diferenciados y opcionales, como alojamientos lujosos en hospitales y tratamientos odontológicos estéticos, estarán disponibles mediante contratación de seguros privados de salud (planes de salud) o pago directo. Sin embargo, los autores resaltan que una razonable participación pública es indispensable en cualquier proceso para establecer un 'mínimo decente' y en la decisión acerca del conjunto de bienes y servicios a ser ofertados (y a ser rechazados) ${ }^{11}$.

De modo diferente, Norman Daniels, en su clásica obra 'Just Health Care'12, busca demostrar la importancia moral de la atención de salud en las políticas sociales y su inserción en las estructuras políticas y económicas de la sociedad, desarrollando una teoría de justicia distributiva aplicable a los cuidados de salud y estableciendo que los principios de su teoría 
son universales (poseen aplicación global), destinándose a las sociedades de todos los niveles de desarrollo, desde países más ricos hasta países más pobres. Norman Daniels establece que una justa distribución de los recursos de salud, aunque en situaciones de escasez, debe ser orientada a reducir las desigualdades en el acceso a la atención de salud mediante el uso de la razonabilidad, preconizando lo que podemos denominar equidad sanitaria.

En otro trabajo, 'Just Health - Meeting Health Needs Fairly'13, Norman Daniels busca responder lo que considera una cuestión fundamental acerca de la justicia para la salud: Por una cuestión de justicia, ¿qué nos debemos unos a los otros para promover y proteger la salud de una población y ayudar a las personas cuando están enfermas o discapacitadas? (As a matter of justice, what do we owe each other to promote and protect health in a population and to assist people when they are ill or disabled?). Para ello, Norman Daniels divide la cuestión fundamental en tres cuestiones focales: 1) La salud y, por lo tanto, la asistencia a la salud y otros factores que afectan la salud $\dot{i}$ tienen especial importancia moral? 2) ¿Cuándo son injustas las desigualdades en salud? 3) ¿Cómo podemos atender las necesidades de salud de forma justa bajo restricciones de recursos?

Respondiendo a las tres preguntas, el autor concluye que la justicia distributiva aplicable a los sistemas de salud debe fundamentarse en la protección de oportunidades abiertas a los individuos (range of opportunities) con la adopción de la justicia como equidad (justice as fairness), mitigando las desigualdades sociales a través de un proceso de toma de decisiones (fair deliberative process), con el fin de garantizar una adecuada distribución de los recursos e igualitario acceso a la atención sanitaria ${ }^{13}$.

Norman Daniels establece como principio adecuado para limitar gastos con la asistencia médica lo que denomina responsabilidad por la razonabilidad (accountability for the reasonableness), lo que promovería una deliberación democrática y justa sobre la utilización de los recursos sanitarios ${ }^{13}$.
Este autor identifica además y enfatiza una profunda e incontestable relación entre las desigualdades sociales y las diversas formas de injusticias, particularmente en el acceso a la atención de salud. También reconoce que tales injusticias pueden ser coyunturales (injusticias domésticas, locales) y estructurales (injusticias internacionales, globales), con profundas y nefastas consecuencias en la limitación del acceso y en la precarización de la calidad de la atención de salud de las poblaciones.

Norman Daniels parte de una visión democrática de justicia social, defendiendo la equidad como una de sus principales características - justicia distributiva que privilegia a los más necesitados y que busca corregir, aunque parcialmente, las desigualdades sociales. Por lo tanto, este autor reconoce el derecho a la salud (aunque limitándose a abordar el derecho de acceso a la atención de salud) y defiende que solo una justa distribución de los recursos sanitarios puede garantizar un sistema de salud igualitario y socialmente justo.

Finalmente, es conveniente aclarar que los autores escogidos encarnan los discursos bioéticos del norte más representativos con relación a la bioética y al acceso a cuidados de salud, no significando esto que no haya otros discursos alternativos en esa parte del mundo, como los de autoras feministas no blancas. Conviene decir, además, que la elección de estos autores apenas obedeció a su audibilidad en el mundo, siendo justificada también por el recorte metodológico (comparativo) al cual e este documento responde.

\section{Los discursos bioéticos del sur}

Los bioeticistas latinoamericanos seleccionados en el presente estudio defienden, de forma complementaria en sus discursos, el derecho a la salud y el acceso universal a la atención de salud, vista como un valor social, un derecho humano fundamental. 
Kottow y Schramm proponen el principio de protección como el más adecuado a los propósitos de una ética de la salud pública, ya que este permitiría una clara identificación de los objetivos y de los actores involucrados con la implementación de políticas públicas moralmente correctas y pragmáticamente efectivas ${ }^{\mathbf{1 4}}$.

En la Bioética de Protección, ampliando los límites de la bioética tradicional, se debe prestar especial atención a los sujetos vulnerables: individuos desprotegidos y desprovistos de condiciones adecuadas de vida, sobre todo en el acceso a la atención de salud ${ }^{15,16}$. Además, la génesis de la Bioética de Protección se vincula al mismo surgimiento del Estado moderno, entendida la protección como una de sus funciones primordiales; un Estado protector, un Estado asistencial, capaz de garantizar las mínimas condiciones de vida para sus ciudadanos, como ingresos, alimentación, vivienda, educación y salud ${ }^{15}$.

Según Schramm, es indiscutible el papel central del Estado como ente responsable por la protección de sus ciudadanos y por la efectividad de políticas públicas de salud que concuerden con el concepto de justicia sanitaria. Esta protección, en el ámbito de las prácticas sanitarias, debe ser considerada a partir de dos vertientes distintas y complementarias la prevención de los agravios a la salud de los individuos (protección negativa) y la promoción del desarrollo humano y de la calidad de vida de las personas (protección positiva) ${ }^{\mathbf{1 7}}$.

En fin, la Bioética de Protección de Kottow y Schramm se configura en una propuesta inclusiva e igualitaria, en que las políticas públicas de salud, por medio de la acción responsable y efectiva del Estado, deben privilegiar a los sujetos vulnerables, siendo la universalidad del acceso a los cuidados de salud una prerrogativa de todos los ciudadanos.

Según Garrafa, la reflexión bioética sobre las cuestiones de salud debe ser ampliada, privilegiando cuestiones colectivas y la esfera pública, más allá de las cuestiones individuales y de la esfera privada ${ }^{\mathbf{1 8}}$. De este modo, Garrafa y Porto, partiendo de una visión latinoamericana de la bioética, proponen una bioética fuerte/dura (hard bioethics) o una Bioética de Intervención (intervention bioethics), en oposición al dominio hegemónico de la bioética principialista estadunidense de Beauchamp y Childress ${ }^{19}$.

Garrafa aclara que la denominada Bioética de Intervención se configura epistemológicamente como una propuesta contundente y crítica frente a la supuesta hegemonía de la bioética principialista, colocándose como una adecuada y necesaria herramienta para sociedades oprimidas con enormes contingentes de individuos y grupos sociales vulnerables, con énfasis en cuestiones sociales, sobre todo en los problemas sanitarios y en defensa de la salud pública ${ }^{20}$.

Este autor resalta a la salud como un importante factor de inclusión social y critica la asimétrica distribución de los recursos y servicios de salud entre ricos y pobres, así como identifica la relevancia del acceso universal a la atención de salud como un compromiso social de gobiernos democráticos, con el fin de garantizar los más altos estándares de salud a sus ciudadanos ${ }^{21}$.

Garrafa, Cunha y Manchola-Castillo ${ }^{22}$, abordando cuestiones éticas en el sistema de salud brasileño (SUS - Sistema Único de Saúde), concluyen que la bioética brasileña no puede acomodarse y debe actuar críticamente en defensa de un sistema público de salud que garantice a sus ciudadanos un acceso universal y equitativo a la asistencia sanitaria.

Se concluye que, a través de su propuesta por la creación de la Bioética de Intervención, Garrafa adopta un discurso directo e intransigente en la defensa de un sistema de salud de acceso universal, desde una perspectiva política y socialmente comprometida con los intereses públicos y los ideales democráticos de justicia social y plena ciudadanía. El autor reconoce el derecho a la salud como una prerrogativa de los individuos en conquistar una vida con calidad y dignidad, adoptando un concepto ampliado de salud como un valor y como un derecho social, que incorpora otros 
derechos humanos fundamentales (individuales, colectivos y difusos).

Así, la Bioética de Intervención se inserta como una propuesta transformadora y emancipadora, en que las políticas públicas de salud, por medio de la acción intervencionista del Estado, deben garantizar la universalidad, la integralidad y la gratuidad de la asistencia a la salud como un derecho humano fundamental y un deber del Estado.

Para concluir este aparte, es conveniente aclarar, tal como manifestado en el anterior, que los autores escogidos encarnan los discursos bioéticos del sur más representativos con relación a la bioética y el acceso a cuidados de salud, no significando esto que no haya otros discursos alternativos en esa parte del mundo, como los de autores latinoamericanos que defienden los postulados de la Organización Mundial de la Salud (OMS). Conviene decir, además, que la elección de estos autores apenas obedeció a su audibilidad y la divergencia de sus posiciones en el mundo, siendo justificada también por el recorte metodológico (comparativo) al que este documento responde.

\section{Consideraciones finales}

Los problemas del acceso a la asistencia sanitaria son comunes en países desarrollados (países ricos, países centrales), y países no desarrollados (países pobres, países periféricos); sin embargo, mientras que entre los mayores desafíos en los primeros aparecen la elevación de los costos con la adopción de nuevas y onerosas tecnologías y la compleja y excesiva burocratización de los sistemas de salud, en los demás, son visibles la precariedad de los servicios derivada de la crónica sub-financiación del sector salud, aliada a permanentes y severos problemas económicos y sociales, como la pobreza, el hambre y el analfabetismo.

Los discursos bioéticos del norte, con fuerte influencia de la ética biomédica, parecen estar más preocupados por la eficiencia financiera de su sistema de salud y con la rentabilidad de las empresas privadas que actúan en el sector, lo que Birn y Hellander denominaron 'market-driven health care' (cuidado de la salud conducido por el mercado) ${ }^{23}$. Por su parte, los discursos bioéticos del sur, representados en las propuestas de las bioéticas críticas aquí expuestas (Bioética de Protección y Bioética de Intervención), con fundamento en los derechos humanos, están comprometidos con cuestiones sociales y en la defensa intransigente de las poblaciones excluidas y marginadas, pauperizadas por acciones predatorias e imperialistas de los países ricos sobre los países pobres.

En este punto, cabe resaltar una importante diferencia entre el acceso universal a la atención de salud, matriz ideológica y ético-política de los sistemas universales de salud (Universal Health System - UHS), y la cobertura universal de salud (Universal Health Coverage - UHC), una propuesta defendida por entidades que representan los intereses de los países ricos y del capital internacional, como el Banco Mundial (BM) (World Bank - WB) y la OMS (World Health Organization - WHO). ${ }^{24}$

El principal objetivo de la UHC es la protección financiera de los individuos contra gastos excesivos con salud, permitiendo que todos puedan acceder a los servicios de salud sin experimentar dificultades financieras. Sin embargo, la denominada 'cobertura' se refiere tan solo a la capacidad de los individuos o las familias para adquirir algún tipo de seguro de salud, lo que no significa garantía de acceso y completa atención de sus necesidades en salud. La dicotomía entre financiamiento y prestación de servicios involucra la fijación de precios de los servicios de salud, transformándolos en mercancía; así, el acceso a la asistencia sanitaria dependerá de las normas de cada seguro de salud y de la capacidad de pago de las personas ${ }^{25}$.

Manchola-Castillo, Garrafa, Cunha y Hellmann26 y Garrafa, Cunha y MancholaCastillo22 afirman que en la mayoría de los casos, la UHC es una falsa propuesta de mejora de las condiciones de salud de las poblaciones, sobre todo perjudicial para los países periféricos, 
pues no pasa de un programa de privatización y tercerización de los sistemas de salud.

A diferencia de lo anterior, el modelo de UHS, cuyos ejemplos más significativos son el sistema nacional de salud del Reino Unido (1948), Portugal (1974), Italia (1978), España (1986) y Brasil (1988) es financiado con recursos públicos, lo que significa una mayor solidaridad, redistribución y equidad, haciendo con que la garantía del acceso universal sea sinónimo de ciudadanía ${ }^{25}$.

En el caso brasileño, desde su implantación a principios de los años 1990, el SUS está basado en una concepción integral de universalidad y de integralidad, permitiendo acceso universal a la atención de salud y asistencia integral de la población, sobre todo a los individuos y familias de bajos ingresos.

En América Latina, actualmente, específicamente en países como Chile, México y Colombia, están en curso diversas reformas en los sistemas nacionales de salud según las directrices propuestas por la UHC (OMS), resultando en diversos problemas para la garantía del acceso a la atención de salud, así como elevación de los costos financieros del sector ${ }^{25}$. En estos casos, se verifica la clara intención del capital financiero internacional para ampliar su participación en el mercado global de seguros y de servicios de salud, a través de empresas aseguradoras y prestadoras de servicios, así como de industrias farmacéuticas y de equipos ${ }^{27}$.

Esta amenaza al derecho universal a la salud también está presente en Brasil, sobre todo después de la reciente apertura del capital y consecuente adquisición de empresas aseguradoras brasileñas por multinacionales extranjeras, con clara pretensión de ampliar su participación en el mercado de salud nacional. Al mismo tiempo, recientes medidas gubernamentales, alineadas con las demandas del sector privado, promueven la reducción del financiamiento público en la salud, con la clara finalidad de debilitar el SUS e incluso extinguirlo o privatizarlo.
Existe una evidente divergencia con respecto al acceso a cuidados de salud entre los discursos del Norte y del Sur globales. Mientras los primeros ven la salud como un producto a ser adquirido, los segundos la consideran un derecho que debe ser garantizado a todos los seres humanos. Más que eso, esta divergencia responde a un contexto económico, político y social particular y, especialmente, a una visión bioética específica, representada en este artículo por Beauchamp, Childress, Engelhardt y Daniels, por un lado, y por Garrafa, Schramm y Kottow, por el otro.

Las dimensiones éticas, políticas, económicas y sociales de la salud la convierten en un importante campo para la reflexión bioética, sobre todo de una bioética que se pretenda crítica y vanguardista, así como preocupada predominantemente con los problemas colectivos y con las necesidades y vicisitudes de las poblaciones más vulnerables. Las políticas de salud pueden promover o violar los derechos humanos, incluyendo el derecho a la salud, dependiendo de cómo se diseñen y se ejecuten. Así, las discriminaciones en el acceso o la prestación de los servicios de salud violan los derechos humanos. La adopción de medidas para garantizar y proteger los derechos humanos es responsabilidad primordial del Estado, en lo que se refiere al sector de salud, a fin de corregir desigualdades, inequidades y prácticas discriminatorias, garantizando salud de calidad para todos.

\section{Colaboradores}

Monteiro PJC (0000-0003-2759-7700)* e Castillo CHM (0000-0003-4084-610X)* participaron en la concepción, planificación, análisis e interpretación de datos; redacción, revisión crítica del contenido y aprobación de la versión final del manuscrito. 


\section{Referencias}

1. Cohen J, Ezer T. Human Rights in Patient Care: a theoretical and practical framework. Health and Human Rights. 2013; 15(2):7-19.

2. Travassos C, Martins M. Uma revisão sobre os conceitos de acesso e utilização de serviços de saúde. Cad. Saúde Pública. 2004; 20(2):190-8.

3. Donabedian A. Aspects of medical care administration: specifying requirements for health care. Cambridge: Harvard University; 1973.

4. Aday LA, Andersen R. A framework for the study of access to medical care. Health Serv Res. 1974; 9(3):208-20.

5. Ronald A, Newman JF. Societal and individual determinants of medical care utilization in the United States. Milbank Mem Fund Q Health Soc. 1973; 51(1):95-124.

6. McIntyre D, Mooney G, editores. The economics of health equity. New York: Cambridge University; 2007.

7. Thiede M, McIntyre D. Information, communication and equitable access to health care: a conceptual note. Cad. Saúde Pública. 2008; 24(5):1168-73.

8. Sanchez RM, Ciconelli RM. Conceitos de acesso à saúde. Rev Panam Salud Publica. 2012; 31(3):260-8.

9. Engelhardt Jr. HT. Fundamentos da Bioética. Tradução de José A. Ceschin. 6. ed. São Paulo: Edições Loyola; 2015.

10. Engelhardt Jr. HT. Fundamentos da Bioética Cristã Ortodoxa. Tradução de Luciana Moreira Pudenzi. São Paulo: Edições Loyola; 2003.

11. Beauchamp T, Childress J. Principles of Biomedical Ethics. 7. ed. Oxford; 2013.

12. Daniels N. Just Health Care - Studies in philosophy and health policy. Cambridge Press; 1985.

13. Daniels N. Just Health - Meeting Health Needs Fairly. Cambridge Press; 2008.
14. Schramm FR, Kottow M. Principios bioéticos en salud pública: limitaciones y propuestas. Cad. Saúde Pública. 2001; 17(4):949-56.

15. Schramm FR. A bioética da proteção em saúde pública. In: Fortes PAC, Zoboli ELCP, organizdores. Bioética e Saúde Pública. São Paulo: Loyola; 2003. p. $71-84$.

16. Schramm FR. Bioética da Proteção: ferramenta válida para enfrentar problemas morais na era da globalização. Bioética. 2008; 16(1):11-23.

17. Schramm FR. A bioética de proteção: uma ferramenta para a avaliação das práticas sanitárias? Ciênc. Saúde Colet. 2017; 22(5):1531-8.

18. Garrafa V. Reflexão sobre políticas públicas brasileiras de saúde à luz da bioética. In: Fortes PAC, Zoboli ELCP, organizadores. Bioética e Saúde Pública. São Paulo: Centro Universitário São Camilo/Edições Loyola; 2003. p. 49-61.

19. Garrafa V, Porto D. Intervention Bioethics: a proposal for peripheral countries in a context of power and injustice. Bioethics. 2003; 17(5-6):399-416.

20. Garrafa V. Da bioética de princípios a uma bioética interventiva. Revista Bioética. 2005; 13(1):125-34.

21. Garrafa V. Ampliação e politização do conceito internacional de bioética. Bioética. 2012; 20(1):09-20.

22. Garrafa V, Cunha TR, Manchola C. Access to healthcare: a central question within Brazilian bioethics. Cambridge Quarterly of Healthcare Ethics. 2018; 27(3):431-9.

23. Birn AE, Hellander I. Market-driven health care mess: the United States. Cad. Saúde Pública. 2016; 32(3):1-4.

24. Organização Mundial de Saúde. Relatório Mundial de Saúde - Financiamento dos sistemas de saúde: o caminho para a cobertura universal. Genebra: OMS; 2010. 
25. Giovanella L, Mendonza-Ruiz A, Pilar ACA, et al. Sistema universal de saúde e cobertura universal: desvendando pressupostos e estratégias. Ciênc. Saúde Colet. 2018; 23(6):1763-76.

26. Manchola-Castillo C, Garrafa V, Cunha T, et al. El acceso a la salud como derecho humano en políticas internacionales: reflexiones críticas y desafíos contemporáneos. Ciênc. Saúde Colet. 2017; 22(7):2151-60.
27. Iriart C, Merhy EE. Inter-capitalistic disputes, biomedicalization and hegemonic medical model. Interface (Botucatu). 2017; 21(63):1005-1016.

Recibido en 02/05/2019

Aprobado en 16/10/2019

Conflicto de intereses: inexistente

Apoyo financiero: no hubo 